\title{
Enforcing Regulatory Standards in Stock Pollution Problems *
}

\author{
Carmen Arguedas ${ }^{\mathrm{a}}$, Francisco Cabo $^{\mathrm{b}}$ and Guiomar Martín-Herrán ${ }^{\mathrm{b}}$ \\ ${ }^{a}$ Dept. Análisis Económico: Teoría Económica e Historia Económica, Universidad Autónoma de Madrid \\ bIMUVa: Dept. Economía Aplicada (Matemáticas), Universidad de Valladolid
}

\begin{abstract}
We analyze optimal pollution standards and enforcement strategies in settings where environmental damages depend on accumulated pollution, and enforcement is socially costly. We assume that a regulator and a representative polluting firm interact in a Stackelberg differential game, and we specifically allow the firm to pollute above the limit, and pay the corresponding fine. A crucial element is how progressive the fine is with respect to the degree of non-compliance. Some of our results contradict the related literature on the control of stock pollutants under full-compliance. In particular, we find that setting standards dependent on the pollution stock (setting quantities) is preferred if fines are sufficiently non-linear; while setting fines dependent on the pollution stock (setting prices) is preferred if fines are linear or almost linear, and specially when the environmental problem is particularly harmful.

Key words: pollution standards; non-compliance; dynamic regulation; Stackelberg differential games: prices versus quantities.

JEL Codes: C61, C73, K32, K42, L51, Q28.
\end{abstract}

\section{Introduction}

Very frequently, emissions released by polluting facilities accumulate overtime and the resulting environmental damages depend on the stock of pollution. Examples include, among others,

*Enforcing Regulatory Standards in Stock Pollution Problems.

Please send all correspondence to: Guiomar Martín-Herrán, Dept. Economía Aplicada (Matemáticas), Facultad de Ciencias Económicas y Empresariales, Universidad de Valladolid, Avda. Valle Esgueva, 6, 47011 Valladolid, Spain. Tel: +34 983 423330. E-mail: guiomar@eco.uva.es

We wish to thank the Associate Editor and one anonymous reviewer for their helpful comments and suggestions, as well as the participants of WCERE (Gothenburg University), VIII AERNA Conference (CSIC, Madrid), Second AERNA Workshop on Game Theory and the Environment (CSIC, Madrid), First Workshop on Economic Theories and Low-Carbon Transformation Policies (University of Cambridge, UK), and research seminars at University of Waseda and NOVA School of Business and Economics. The authors acknowledge financial support from the Spanish Government under research projects ECO2017-82449-P (Carmen Arguedas), and ECO2014-52343-P and ECO2017-82227-P (Francisco Cabo and Guiomar Martín-Herrán), as well as financial aid from Junta de Castilla y León VA024P17 and VA105G18, co-financed by FEDER funds (Francisco Cabo and Guiomar Martín-Herrán). 
global warming and wastewater discharges. So far, the literature has offered the modelling of optimal environmental policies in such dynamic contexts under alternative assumptions, for example, in cases of asymmetric information between regulators and polluting sources (see, for example, Hoel and Karp, 2001, and the references cited therein), when firms undertake investment decisions that affect future abatement costs (Biglaiser et al., 1995, Karp and Zhang, 2016), or in non-point pollution problems with heterogeneous sources (Xabadia et al., 2008, Goetz and Xabadia, 2015). However, all these studies acknowledge full compliance.

In this paper, we deal with the possibility that polluting sources deliberately exceed the regulations in stock pollution problems. For example, in the case of pollution limits or standards, this occurs when firms decide to pollute above the limits. This may be the case when the marginal expected costs of violating the standard (or the expected fine for non-compliance) is lower than the marginal abatement cost savings of exceeding it.

An important environmental problem where our analysis can be applied is the pollution of rivers and lakes from industrial saline wastewater discharges. Many point sources are likely to generate highly saline wastewater, including agro-food, petroleum, textile, leather, mining or power industries, see, for example, Lefebvre and Moletta (2006). These discharges are known to adversely affect aquatic life, water potability and agriculture. Most legislations around the world, such as the Water Framework Directive 2000/60/EC, the US Clean Water Act 1972, or the Australian legislation, establish measures to prevent the adverse impacts from saline pollution. In practice, restrictions of wastewater discharges are regulated by means of pollution permits that specify maximum allowed levels of salt concentration at each specific point source, depending on the backround level of salt in the river.

An example in point is the regulation of saline pollution of the Hunter River, in Australia. ${ }^{1}$ The Hunter River contains high levels of salt as a result of run-off and infiltration, weathering of the geological strata, and saline groundwater inflows, but also due to a range of anthropogenic sources, such as mining, land clearing and agriculture. The regulation balances the water quality needs of agricultural users with the discharge needs of mines and power stations. Facilities use permits to discharge their salty water into the river, but only when it contains adequate fresh water to dilute the salt and maintain water quality. Therefore, the scheme balances the amount of salt that industries can directly discharge with the background level of salt in the river. When river flows are low, no discharges are allowed. During periods of high flows, limited discharges can occur; but only if facilities have sufficient salt credits. The scheme is operated by WaterNSW under a service agreement with the NSW Environment Protection Authority (EPA).

\footnotetext{
${ }^{1}$ We are very grateful to an anonymous referee for suggesting this example. Detailed information can be found at https://www.epa.nsw.gov.au/licensing-and-regulation/licensing/environment-protectionlicences/emissions-trading/hunter-river-salinity-trading-scheme
} 
To ensure compliance with the permits, the program uses a broad range of enforcement mechanisms, including monetary penalties, formal warnings, official cautions, licence conditions, notices, directions, and prosecutions. ${ }^{2}$ However, these enforcement tools do not ensure full compliance. Indeed, several companies have been alleged by EPA to have breached environmental legislation. ${ }^{3}$

This example illustrates the importance of studying pollution standards and enforcement strategies in stock pollution problems, taking into account the possibility that facilities may decide to exceed the standards. This is our objective in this paper. We specifically model the dynamic interaction between a representative polluting firm and a regulator by means of a differential game played à la Stackelberg. Firm's emissions accumulate overtime, and environmental damages at a given time depend on the pollution stock. To correct for the externality, the regulator sets a pollution standard and a fine for non-compliance overtime, while the firm chooses emissions, which could be, at any time, above the standard. We assume that the firm seeks to maximize discounted profits throughout an infinite time horizon, while the regulator is concerned about social welfare, that is, firm's profits net of environmental damages and the social costs of implementing sanctions.

In our context of non-compliance, it is important to point out that the regulator controls two instruments simultaneously: the pollution standard (quantity instrument) and the fine for non-compliance (price instrument). Put differently, the standard cannot be enforced without a fine. This clearly contrasts with previous literature on the control of stock pollution under full compliance, where the relative merits of either a standard or a tax are analyzed. There, either a standard is imposed, and firms are assumed to pollute exactly what they are commanded without paying anything; or a tax per unit of pollution is imposed, and firms decide (and pay for) all pollution discharges. Importantly, while our fine is clearly a price instrument, however, it should not be exactly referred to as a tax, since it only applies for pollution levels in excess of the standard. The firm will not pay any amount as long as it matches the standard; and it will only pay for discharges in excess of the standard. ${ }^{4}$

Our analysis considers subgame-perfect and hence time-consistent regulatory policies which, in consequence, are credible policies. Interestingly, the optimal standard becomes harsher as

${ }^{2}$ For more information about EPA's regulatory tools, see the EPA Compliance Policy at https://www.epa.nsw.gov.au/licensing-and-regulation/legislation-and-compliance/policies-and-guidelines

${ }^{3}$ At https://www.epa.nsw.gov.au/licensing-and-regulation/public-registers/about-prpoeo/enforceableundertakings-media-releases, there is plenty of information about many recent enforceable undertakings (voluntary, legally binding, written agreements between the EPA and companies in response to alleged breaches), designed to improve environmental outcomes and prevent similar breaches occurring in the future. This information suggests that non-compliance within the scheme is not anecdotal.

${ }^{4}$ More precisely, the fine and the tax are equivalent only when the standard is zero. 
long as the environmental problem worsens overtime, while the optimal fine becomes larger. Thus, both the standard and the fine work as complementary instruments for pollution control, and become harsher (softer) as long as the pollution stock increases (decreases). ${ }^{5}$

From this benchmark scenario where the regulator can control both the standard and the fine overtime (scenario $Q F$ ), we then move to analyze other more restrictive, although probably more realistic, situations. One possibility is that the regulator sets the emissions standard overtime, while the fine is an exogenous constant. This is our scenario $Q$ (or quantity scenario). An alternative situation occurs when the regulator only sets the fine, while the emissions standard is an exogenous constant (scenario $F$, or price scenario). ${ }^{6}$

In these two scenarios $Q$ and $F$, we find that the respective control variables evolve in a similar fashion as in the benchmark case $Q F$ described above. That is, the standard decreases as the pollution stock increases (in scenario $Q$ ), while the fine for non-compliance increases as the pollution stock increases (in scenario $F$ ). Interestingly, scenario $Q$ approaches scenario $Q F$, as long as the progressive part of the fine for non-compliance is more important, while the performance of scenario $F$ is much less dependent on this progressive term.

A relevant research question in all this constrained analysis is What type of regulation is preferred when non-compliance is an issue? Should we pick scenario $Q$ or scenario $F$ ? Put differently, should the regulation be based on quantities or on prices? In a static context of asymmetric information, the seminal paper by Weitzman (1974) shows that regulating through quantities is preferred as long as marginal environmental damages are steeper than marginal abatement costs. Later on, Hoel and Karp (2001) extend this analysis to stock pollution problems, showing that a steeper marginal environmental damage, a lower decay rate or a higher discount factor (all of them suggesting that the environmental problem worsens) favor the use of quantities. The first effect matches the result by Weitzman (1974), while the other two are specific of stock pollution problems.

The question here is whether we can make a similar claim when non-compliance is an issue.

\footnotetext{
${ }^{5}$ Some empirical works, such as Earnhart (1997), Kleit et al. (1998), Oljaca et al. (1998) or Billiet et al. (2014) among others, find that in practice fines increase with the harm caused by the violation (see also Rousseau, 2007, for an overview of the empirical literature on the determinants of the sanctions for environmental offenses). However, we are not aware of any other theoretical study that raises this issue.

${ }^{6}$ Both scenarios could, in principle, illustrate realistic regulatory settings. Going back to our example on saline wastewater, scenario $Q$ can reflect the setting, at the local level, of more stringent or laxer standards, depending on the particular background level of salt in the river, while fines for non-compliance are set at the federal level and do not take the pollution stock into account (maybe because this information is not readily accesible for the government body responsible for setting the fines, or because there is heterogeneity in the specific circumstances of the different point sources along the river course). Alternatively, scenario $F$ can illustrate a case where standards are decided at the upper level (and they are not contingent on the stock of salt), while the enforcement aspects (the fine, but also the monitoring) are decided at the local level, such that the expected fine becomes more or less stringent depending on the specific gravity of the problem at a specific point source.
} 
Importantly, our focus is different: we concentrate on the effects of non-compliance, while at the same time we assume symmetric information between the regulator and the firm. ${ }^{7}$ This implies perfect monitoring, which helps us to concentrate on the effects of the penalties for non-compliance. ${ }^{8}$

An idiosyncratic aspect in our study is the specific structure of the fine for non-compliance. For tractability reasons, we consider a linear-quadratic shape, where the regulator can only control the linear part. ${ }^{9}$ There is, however, an additional quadratic component that measures the progressivity of the fine, which is crucial in the performance of all scenarios, specially in scenario $Q$. As long as the quadratic component of the fine increases, scenario $Q$ becomes more attractive than scenario $F$. The reason is that the standard becomes more effective in affecting pollution, since the marginal fine depends on the standard, and this dependence increases with the progressivity of the fine. ${ }^{10}$

Our results suggest that controlling through fines (prices) is preferred under linear enforcement, while controlling through standards (quantities) is preferred under progressive enforcement (and the more progressive the fine, the more in favor of standards). These results are robust to different specifications of the most relevant parameters of the problem, namely environmental damages, the discount rate, or the degree of assimilative capacity of the environment. When the fine is sufficiently progressive, the prevalence of quantities (scenario $Q$ ) is preserved, regardless of the specific parameters describing the environmental problem. However, as long as the fine is not that progressive, larger environmental damages, lower discount rates or lower decay rates favor the use of prices (scenario $F$ ). Again, scenario $Q$ is not relevant for controlling emissions when the fine is linear or not sufficiently progressive, and therefore scenario $F$ gains importance in controlling harmful situations.

Hence, our different focus results in a different conclusion. As pointed out above, Hoel and Karp (2001) consider asymmetric information while they acknowledge full compliance, and they conclude that quantities are preferred under larger environmental damages, lower discount rates or lower decay rates. In our context, however, non-compliance is an issue and information

\footnotetext{
${ }^{7}$ By symmetric information we mean that, with no uncertainty, all players have complete information, although the game is played hierarchically à la Stackelberg.

${ }^{8}$ Under imperfect monitoring, we would need to consider a third regulatory instrument in the analysis, the inspection probability, that would complicate matters unnecessarily. Alternatively, in a context of risk neutrality, we can think that all the analysis about the fine is indeed an analysis of expected fine (that is, inspection probability times the fine), where the explicit determination of the inspection probability is embedded in the determination of the expected penalty.

${ }^{9}$ This only applies in scenarios $Q F$ and $F$. In scenario $Q$, the regulator cannot control the fine at all.

${ }^{10}$ Under linear enforcement, the marginal fine is a constant. As a result, the specific level of the standard cannot affect pollution, and the standard itself cannot affect the dynamics of the problem.
} 
problems are absent, and we find the prevalence for prices in the cases where the environmental problem is harmful (as long as fines are not very progresive in the amount of the violation).

Besides scenarios $Q$ and $F$, a second set of restrictions entails situations where agents partially ignore the pollution stock. On the one hand, we assume that the regulator can control the two instruments (standard and fine), but he/she is restricted to constant values of the standard and the fine. These constraints may obey practical reasons dealing with simplicity of the regulation, for example. However, a more simplistic implementation comes at the cost of lower credibility, since these policies are no longer time-consistent. On the other hand, we also consider the case where the firm behaves myopically, not acknowledging that higher pollution will induce a higher fine and a lower standard. In the myopic case, policies are credible and the regulator needs to rely on harsher fine and standard. Both cases result in higher long-run pollution stock and lower social welfare.

Up to our knowledge, this is the first theoretical study of this kind. As stated before, several papers analyze optimal regulations in dynamic settings, but they do so by assuming perfect compliance. ${ }^{11}$ Also, the vast majority of existing works about the relationship between regulatory stringency and compliance assumes static contexts, and therefore neglects the possibility that pollution may accumulate overtime, see, for example, Downing and Watson (1974), Harford (1978), Jones and Scotchmer (1990), Keeler (1995), or Arguedas (2008, 2013), among others. There is also a relevant literature on optimal dynamic enforcement, for example, Harrington (1988), Harford and Harrington (1991), Raymond (1999), Friesen (2003), or Zhang and Xu (2016), but these studies assume exogenous standards. Up to our knowledge, the only exception that studies optimal standard setting in dynamic contexts allowing for non-compliance is Arguedas et. al (2017a), but restricted to flow pollution contexts.

The remainder of the paper is organized as follows. In Section 2 we present the model. In Section 3, we analyze the characteristics of the benchmark policy where the regulator can control for both the pollution standard and the fine (scenario $Q F$ ). In Section 4, we extensively compare this scenario with two restrictive situations where the regulator can only control for the standard being the fine exogenous (scenario $Q$ ), or the fine, being the standard exogenous (scenario $F$ ). In Section 5, we present other restrictive cases where, for several reasons, decision agents may partially ignore the pollution stock. We conclude in Section 6. All the technical details and some complementary material can be found in an Appendix available online.

\footnotetext{
${ }^{11}$ Additional references are Beavis and Dobb (1986), Hartl (1992), Conrad (1992), Falk and Mendelsohn (1993) or Benford (1998).
} 


\section{The model}

A representative firm produces a consumption good with emissions as the only input. Let $Y(t)$, $E(t)$ respectively denote production and emissions of the facility at time $t$. For mathematical convenience, we assume that the value of production is linear-quadratic in emissions, as follows:

$$
Y(E(t))=\sigma E(t)-\frac{E(t)^{2}}{2} .
$$

Thus, production is strictly concave in emissions, and it is maximum for $E(t)=\sigma$.

The flow of emissions is the only decision variable of the firm. Emissions accumulate overtime as a stock of pollutants, $P(t)$, according to the dynamic rule:

$$
\dot{P}(t)=E(t)-\delta P(t), \quad P(0)=p_{0},
$$

where $\delta>0$ is the degree of assimilative capacity (or decay rate) of the environment, and $p_{0} \geq 0$ is the stock of pollution at $t=0$.

From the point of view of the firm, emissions are free in the absence of any regulation. However, the stock of pollution causes environmental damages, given by the expression $D(P(t))=$ $d(P(t))^{2} / 2$, with $d>0 .{ }^{12}$

We assume that an environmentally concerned regulator seeks to make the firm (partially) responsible for the environmental damages caused. To internalize the environmental damage, the regulator imposes an emission target or pollution limit which can vary across time, $Q(t)$. Assuming that the regulator can observe emissions $E(t)$ at no cost, the firm faces at each time $t$ the following fine if it is found exceeding the standard, that is, if $E(t)>Q(t)::^{13}$

$$
G(E(t), Q(t), F(t))=F(t)[E(t)-Q(t)]+\theta \frac{[E(t)-Q(t)]^{2}}{2} .
$$

The fine for non-compliance has two components, a linear and a quadratic (progressive) component. For presentation purposes, we refer to $F(t)$ as the fine per unit of the violation (although obviously, the fine is not proportional as long as $\theta>0$ ). This term $F(t)$ can in principle change overtime. For tractability reasons, parameter $\theta \geq 0$ is assumed to be constant and exogenous in the problem. This parameter reflects the importance of the progressive part of the fine.

\footnotetext{
${ }^{12} \mathrm{~A}$ linear quadratic production function in emissions, a linear dynamics for the pollution stock and a quadratic damage function, are common assumptions in dynamic games settings (see, for example, Jørgensen et al., 2010).

${ }^{13}$ Nothing in this expression prevents the possibility that the firm can pollute below the standard and obtain a subsidy for over-compliance. We take this approach for tractability considerations, although we will specifically exclude these cases from the analysis later on, in accordance with most regulations in practice.
} 
In the benchmark scenario (called scenario $Q F$ later on), the environmental regulation is composed of two instruments: the emissions standard, $Q(t)$, and the fine per unit, $F(t)$. In more restrictive (although more realistic) regulatory settings, though, the regulator may be able to choose the standard or the fine only, or restrict him/ herself to constant instruments overtime, as we will present below.

The interaction between the regulator and the firm is described as a Stackelberg differential game in which the regulator is a leader and the firm is a follower. In order to have a timeconsistent solution, we assume that the regulatory policy is set and announced by the leadingregulator as dependent on the actual stock of pollution, $P$. Correspondingly, the follower-firm chooses emissions, taking the regulatory policy and the pollution stock into consideration.

We consider a stagewise feedback Stackelberg solution. Thus, we analyze a differential game played à la Stackelberg in feedback strategies, where the regulator has a stagewise firstmover advantage, i.e. an instantaneous advantage at each time. This solution satisfies subgame perfectness which is the strongest credibility concept. As it is usual for this type of differential games with an infinite time horizon, we assume that agents (the firm and the regulator) employ stationary strategies, i.e, their strategies and value functions do not explicitly depend on time, but exclusively on the pollution stock (see, for example, Haurie et al. 2012).

To characterize the stagewise feedback Stackelberg solution, the firm chooses emissions to maximize the present value of profits over an infinite time horizon, taking the time evolution of the pollution stock into account. Instantaneous profits are given by the income from production, minus the fine for non-compliance. Considering the consumption good as the numéraire, the dynamic maximization problem for the firm is: ${ }^{14}$

$$
\begin{aligned}
& \max _{E} \int_{0}^{\infty}[Y(E)-G(E, Q, F)] e^{-\rho t} d t \\
& \text { s.t.: } \dot{P}=E-\delta P, \quad P(0)=p_{0},
\end{aligned}
$$

where $\rho>0$ is the discount factor, $Y(E)$ and $G(E, Q, F)$ are respectively given by (1) and (3), and $(Q, F)$ are chosen and announced by the regulator to the firm. The firm's optimal response function is given by $\widehat{E}(P ; Q, F)$. Therefore, emissions are a function of the state variable, $P$, and the regulatory variables, $(Q, F)$.

The regulator decides upon the optimal regulatory instruments, taking into account the firm's best response, $\widehat{E}(P ; Q, F)$. Assuming a feedback information structure, strategies are settled by the regulator as functions of the pollution stock $Q(P), F(P)$. The regulator is concerned about

\footnotetext{
${ }^{14}$ The time argument is omitted here and henceforth when no confusion can arise. As a general principle, uppercase letters denote time-dependent (either state or control) variables, while lower-case and Greek letters denote time-independent parameters.
} 
firm's profits, environmental damages, and the social costs of assesing fines, or sanctioning costs. We consider the following quadratic expression for the latter:

$$
H(E, Q, F)=\beta \frac{(E-Q)^{2}}{2}+\xi\left(\frac{F^{2}}{2}+\frac{\theta^{2}}{2}\right),
$$

with $\beta \geq 0$ and $\xi \geq 0$.

The first component of expression (5), $\beta(E-Q)^{2} / 2$, may account, for example, for the administrative costs of court processes. In our context, fines are linear-quadratic in the degree of non-compliance. It seems natural to assume that the associated sanctioning costs will be quadratic as well. Also, the media can contribute to influence citizens' discomfort with large transgressions, which may also justify the quadratic dependence of the sancioning costs on the degree of non-compliance. ${ }^{15}$ The second component of expression (5) may reflect the general discomfort in society with large fines (expressed both in large $F$ and large $\theta$ ). For example, firms may lobby the imposition of large fines, and this may result in additional implementation costs. ${ }^{16}$ Throughout the paper we assume that $\beta>\theta$, which means that the marginal fine for the firm associated with non-compliance is smaller than the marginal administrative costs for the regulator. ${ }^{17}$

Taking into account the firm's optimal response function, $\widehat{E}(P ; Q, F)$, the regulator faces the following dynamic problem:

$$
\begin{aligned}
& \max _{Q, F} \int_{0}^{\infty}[Y(\widehat{E}(P ; Q, F))-D(P)-H(\widehat{E}(P ; Q, F), Q, F)] e^{-\rho t} d t, \\
& \text { s.t.: } \dot{P}=\widehat{E}(P ; Q, F)-\delta P, \quad P(0)=p_{0} .
\end{aligned}
$$

Once the optimal regulatory instruments $Q$ and $F$ are determined, the corresponding firm's optimal emissions and the resulting optimal time-path of the pollution stock can be obtained. In the next section, we present the features of the benchmark policy.

\footnotetext{
${ }^{15}$ Polinsky and Shavell (1992), Stranlund (2007) or Arguedas (2008, 2013) also consider sanctioning costs dependent on the level of non-compliance.

${ }^{16}$ This is similar to Chen and Lai (2012), who analyze the effects of lobbying on the stringency of an emission tax; or more recently, Arguedas et. al (2017b), who investigate the influence of interest groups on the stringency of an emissions standard.

${ }^{17}$ This is the interesting case for our purposes, not only because we aim to compare the case of a linear fine $(\theta=0)$ with non-linear cases with relatively small progressivity, but also because, as we will see later on, this assumption leads to the intuitive result of complementary between the fine and the standard in reducing emissions. The alternative assumption with $\beta<\theta$ results in the two instruments being substitutes.
} 


\section{The Benchmark Policy}

In this section, we present the main characteristics of the solution to problem (6). This baseline scenario is referred to as scenario $Q F$, since the regulator has the capacity to control both the pollution standard and the fine for non-compliance overtime. All the technical details, including a sketch of the analytical treatment of a stagewise feedback Stackelberg equilibrium, are in online Appendix A. For presentation purposes, the analysis is essentially carried out numerically, considering the following set of parameters values: ${ }^{18}$

$$
\sigma=10, \quad \rho=0.03, \quad \delta=0.1, \quad d=0.05, \quad \xi=1, \quad \beta=2, p_{0}=10
$$

Nevertheless, we first present some general properties of the benchmark scenario, which may help us to understand the numerical analysis later on. The firm is a follower, so we first solve problem (4). The firm chooses emissions as a function of the stock of pollution and the regulatory policy as follows:

$$
\widehat{E}^{Q F}(P ; Q, F)=\frac{\sigma+\theta Q-F+\left(V^{Q F}\right)^{\prime}(P)}{1+\theta},
$$

where $V^{Q F}(P)$ is the firm's value function. Note that the standard only influences firm's emissions if the fine is progressive in the degree of non-compliance, that is, if $\theta>0$. Emissions are positively affected by the standard, the maximum marginal productivity of emissions and the firm's negative valuation of the stock of pollution, and they are negatively affected by the per-unit fine for non-compliance.

Next, the regulator maximizes problem (6) taking the firm's best-reaction function (8) into account. Thus, from the first order optimality conditions (available in the Appendix), the optimal feedback strategies are given by:

$$
\begin{aligned}
& Q^{Q F}(P)=\sigma+\frac{\xi(\beta-\theta)\left(V^{Q F}\right)^{\prime}(P)+(\beta+\theta(1+\theta) \xi)\left(W^{Q F}\right)^{\prime}(P)}{\beta+\left(\beta+\theta^{2}\right) \xi}, \\
& F^{Q F}(P)=\beta \frac{\left(V^{Q F}\right)^{\prime}(P)-\left(W^{Q F}\right)^{\prime}(P)}{\beta+\left(\beta+\theta^{2}\right) \xi}
\end{aligned}
$$

where $W^{Q F}(P)$ represents the regulator's value function. The optimal limit, the fine and the emissions stemming from expressions (8), (9) and (10) are defined as functions of the pollution stock leading to a subgame-perfect equilibrium. From (8) and (9) it follows that $E^{Q F}(P)-$ $Q^{Q F}(P)=\theta \xi F^{Q F}(P) / \beta$. In consequence, as long as the optimal fine is positive, the firm

\footnotetext{
${ }^{18} \mathrm{We}$ have carried out an extensive analysis of the robustness of the results to changes in the main parameters of the problem, available from the authors upon request,
} 
exceeds the standard. By contrast, the particular case of a linear fine, $\theta=0$, always yields full compliance. ${ }^{19}$

Our numerical simulations are consistent with the expected result $\left(W^{Q F}\right)^{\prime}(P)<\left(V^{Q F}\right)^{\prime}(P)<$ 0 , meaning that a marginal rise in pollution is more negatively valued by the regulator than by the firm. Furthermore, the emissions limit decreases and the fine per unit increases with the pollution stock. Thus, the worse the environmental problem, the more stringent the environmental policy (both the fine and the limit), and the lower the induced emissions.

\section{Constrained solutions I: The regulator has access to only one instrument}

In this section, we study more restrictive, although probably more realistic settings where the regulator is able to choose only one instrument, either the standard or the fine per unit. The other instrument is exogenously imposed by a third party, for example, a different layer of the Government. In this context, the regulator looses flexibility, as he/she cannot choose or adapt one of the two enforcement mechanisms to the changing environment.

In the first scenario, the regulator takes the fine per unit as given $(\bar{F})$, and determines the optimal standard as a function of the pollution stock (this is scenario $Q$, or the quantity scenario). Alternatively, in the second scenario, the regulator takes the standard as given $(\bar{Q})$, and chooses the fine per unit as a function of the pollution stock (this is scenario $F$, or the price scenario).

Our objective is to analyze which of the two scenarios performs better in social welfare terms. The analysis is complicated because mainly due to coordination failures, the value of the exogenous instrument in each respective scenario may or may not be at its efficient level (that is, the level that maximizes the regulator's value function). We first comment on the general features of the two constrained scenarios (the technical details being in the Appendix), and we then discuss on the main findings.

\subsection{Scenario $Q$}

The firm's best-reaction function when the regulator exclusively chooses the standard is given by $\widehat{E}(P ; Q, \bar{F})$, which corresponds to expression (8) with $F=\bar{F}$. For given $\bar{F}$, the optimal

\footnotetext{
${ }^{19}$ In this particular case, the marginal fine is constant and firm's emissions are not affected by the emissions standard. Thus, the regulator does not use the standard to induce emissions reductions, but to minimize administrative costs at the level anticipated from (8). In consequence, this case always yields full-compliance, $E^{Q F}(P)=Q^{Q F}(P)$ for any stock of pollution.
} 
standard is:

$$
Q^{Q}(P)=\sigma-\frac{(\beta-\theta) \bar{F}-(\beta-\theta)\left(V^{Q}\right)^{\prime}(P)-\theta(1+\theta)\left(W^{Q}\right)^{\prime}(P)}{\beta+\theta^{2}} .
$$

Note that the standard is equally affected by the fine per unit, $\bar{F}$, and the firm's negative valuation of the stock of pollution, $\left(V^{Q}\right)^{\prime}(P)$. Since $\beta>\theta$, either a stricter fine or a higher self-restraint of the firm will directly induce a reduction in the firm's emissions, as shown in expression (8). However, the reduction planned by the firm is too small from the regulator's viewpoint. For that reason, the regulator induces further emissions reductions by tightening the standard. Thus, the exogenous fine $\bar{F}$ and the chosen standard, $Q^{Q}(P)$, are complementary instruments. Likewise, the more environmentally concerned the firm is, the tougher the emissions standard. ${ }^{20}$

From the emissions standard, firm's optimal emissions are:

$$
E^{Q}(P)=\sigma-\frac{\beta \bar{F}-\beta\left(V^{Q}\right)^{\prime}(P)-\theta^{2}\left(W^{Q}\right)^{\prime}(P)}{\beta+\theta^{2}} .
$$

It is easy to see that this scenario generally results in non-compliance, except in the particular case of a linear fine, $\theta=0$. The question is which level of $\bar{F}$ to consider. One reasonable possibility is to determine the value leading to the highest social welfare, named the efficient fine, $\bar{F}^{*}{ }^{21}$ Whether the governmental unit responsible for setting the constant fine correctly settles $\bar{F}^{*}$ or deviates from it importantly affects the results, as we will see later on. For completeness, we consider values of $\bar{F}$ ranging from 0 to the threshold where emissions match the first-best values (the case of a maximizing firm which fully internalizes environmental damages), which always results in full compliance. ${ }^{22}$. Thus, any lower level of the per-unit fine (including the efficient level $\bar{F}^{*}$ ) results in non-compliance.

\subsection{Scenario $F$}

When the regulator exclusively chooses the fine per unit, the firm's best reaction is given by $\widehat{E}(P ; \bar{Q}, F)$ (expression $(8)$ with $Q=\bar{Q})$. For given $\bar{Q}$, the optimal fine and induced emissions

\footnotetext{
${ }^{20}$ This case where the two regulatory instruments are complementary is intuitive and also interesting for our purposes, since we aim to compare the linear case $\theta=0$ with non-linear cases with relatively small progressivity. In the alternative situation with $\beta<\theta$, the fine and the standard would be substitutes in reducing emissions.

${ }^{21}$ Plugging $E^{Q}(P)$ in (11) into (2), we can obtain the optimal time path of the pollution stock as a function of $\bar{F}$. Thus, social welfare in (6) can also be computed as a function of $\bar{F}$, and $\bar{F}^{*}$ is the value that maximizes this function.

${ }^{22}$ We do not study values of $\bar{F}$ above this threshold, since this would lead to a steady-state pollution stock below its first-best value, which would represent an over-preserved environment.
} 
are:

$$
\begin{aligned}
& F^{F}(P)=\frac{(\beta-\theta)(\sigma-\bar{Q})+(1+\beta)\left(V^{F}\right)^{\prime}(P)-(1+\theta)\left(W^{F}\right)^{\prime}(P)}{1+\beta+(1+\theta)^{2} \xi} \\
& E^{F}(P)=\frac{(1+(1+\theta) \xi) \sigma+\theta(1+(1+\theta) \xi) \bar{Q}+(1+\theta) \xi\left(V^{F}\right)^{\prime}(P)+\left(W^{F}\right)^{\prime}(P)}{1+\beta+(1+\theta)^{2} \xi} .
\end{aligned}
$$

Again, $\bar{Q}$ and $F^{F}(P)$ are complementary under the assumption $\beta>\theta$. In this scenario $E^{F}(P)-\bar{Q}$ is generally different from zero even in the case of the linear fine, $\theta=0$. The efficient standard, $\bar{Q}^{*}$, can be characterized following a similar reasoning as in scenario $Q$, see footnote 21 . Likewise, the exogenous $\bar{Q}$ can be adequately placed at the efficient level $\bar{Q}^{*}$ or not. At the steady state, depending on $\bar{Q}$, we can observe situations of under-, full- or overcompliance. The threshold of the emissions limit that ensures full compliance is $\tilde{\bar{Q}}=E^{F}(P)$. In the long run, the firm over-complies at larger values, and it does not comply at lower values of the standard. Although for symmetry with scenario $Q$ (and also completeness), we analyze all the cases of $\bar{Q}$ ranging from 0 to the threshold that induces emissions to match the first-best values. however, we will concentrate on the cases that induce non- or full-compliance. ${ }^{23}$

\subsection{Discussion}

In this subsection, we compare the two constrained policy scenarios described above between themselves, and also with respect to the baseline scenario $Q F$ presented in Section 3. First, we analyze the evolution of the main variables (pollution stock, emissions, policy instruments and degree of non-compliance) for the three scenarios overtime. In this analysis we assume that the corresponding exogenous instrument of each respective scenario is always at its efficient level ( $\bar{F}^{*}$ or $\bar{Q}^{*}$, as described above). Then, we analyze the performance of the different scenarios in terms of social welfare. Obviously, the two constrained scenarios are worse than the benchmark scenario $Q F$ in this respect, so the comparison is essentially carried out between scenarios $Q$ and $F$. As the analysis will show, this comparison crucially depends first on whether and to what extent the exogenously imposed instrument fails to hit its efficient level; and second, on how important the progressive part of the fine is.

Figure 1 represents, in rows, the evolution of the pollution stock, emissions, fine per unit, standard, and degree of non-compliance, under the three different scenarios. ${ }^{24}$ Column 1 refers

\footnotetext{
${ }^{23}$ Situations of over-compliance are technically possible in our context, but regulators might be willing to avoid them due to potential political problems associated with polluters receiving subsidies instead of paying fines.

${ }^{24}$ For the parameter values presented in (7), in scenario $Q$ the efficient fine is $\bar{F}^{*}=5.97375$ (for $\theta=0.1$ ) and $\bar{F}^{*}=4.62299$ (for $\theta=0.4$ ). Likewise, in scenario $F$, the efficient standard is $\bar{Q}^{*}=2.02718$ (for $\theta=0.1$ ) and $\bar{Q}^{*}=1.22156$ (for $\left.\theta=0.4\right)$.
} 
to a mild progressivity of the fine, $\theta=0.1$, while a stronger progressivity, $\theta=0.4$, is presented in column 2.

Given an initially small level of the pollution stock, the three policy scenarios induce an increase in the pollution stock towards a steady state below the laissez-faire long-run pollution stock. As Figures in rows 1, 3 and 4 show (both left and right), the worse the environmental problem, the tighter the fine per unit or the emission limit (in the scenarios where the respective instrument can be chosen). Emissions progressively decrease overtime, since the fine per unit increases overtime (in the scenarios where the fine is a decision variable) and the standard decreases overtime (in the scenarios where the standard is a decision variable). Row 3 in Figure 1 shows that the fine per unit is initially lower under scenario $F$, but after some time it becomes larger than its constant efficient level, $\bar{F}^{*}$. Correspondingly, row 4 shows that when the regulator controls the emissions standard, this is always laxer than its constant efficient level, $\bar{Q}^{*}$. Finally, the level of non-compliance decreases in scenario $F$, as the fine rises, while it increases in scenario $Q$ even though the limit tightens.

While all these properties remain valid both at the left and right parts of Figure 1, the effect of a more progressive fine (higher $\theta$ ) can be deduced moving from left to right. In scenario $Q$, the larger the progressivity of the fine, the closer is the standard to the one set under scenario $Q F$. Likewise, emissions and the stock of pollution under scenario $Q$ evolve more closely to those under scenario $Q F$, for a larger $\theta$. Row 5 also shows that the increase in the level of noncompliance when the standard is optimally chosen is more pronounced the greater the value of $\theta .^{25}$

All this analysis can be summarized as follows:

Claim 1 The more progressive the fine for non-compliance (larger $\theta$ ), the more effectively scenario $Q$ achieves emissions reductions. The standard, the emissions and the pollution stock under scenario $Q$ are closer to the benchmark scenario $Q F$ as long as $\theta$ increases.

These results suggest that a more progressive fine favors a regulation through quantities than through prices. The explanation relies on the different roles that the standard and the perunit fine play in the marginal fine for non-compliance, as long as the progressive component $\theta$ increases. ${ }^{26}$ All this is reinforced with the help of Figure 2, where we depict the social welfare

\footnotetext{
${ }^{25}$ This reflects the fact that the emissions limit does induce emissions reductions when $\theta>0$, contrary to the linear case, $\theta=0$. In this latter case, the standard only aims at attaining full-compliance and hence minimum administrative costs for the regulator.

${ }^{26}$ Given the expression for the fine $(3)$, the additional fine for an extra unit of non-compliance (everything else equal) is $F+\theta(E-Q)$. Both instruments $F$ and $Q$ are in this expression (except in the linear case, where $Q$ does not affect the marginal fine). However, as long as $\theta$ increases, $Q$ becomes more important, in fact it is the only instrument remaining in the expression for the cross effect w.r.t. $\theta$, which results in $(E-Q)$.
} 

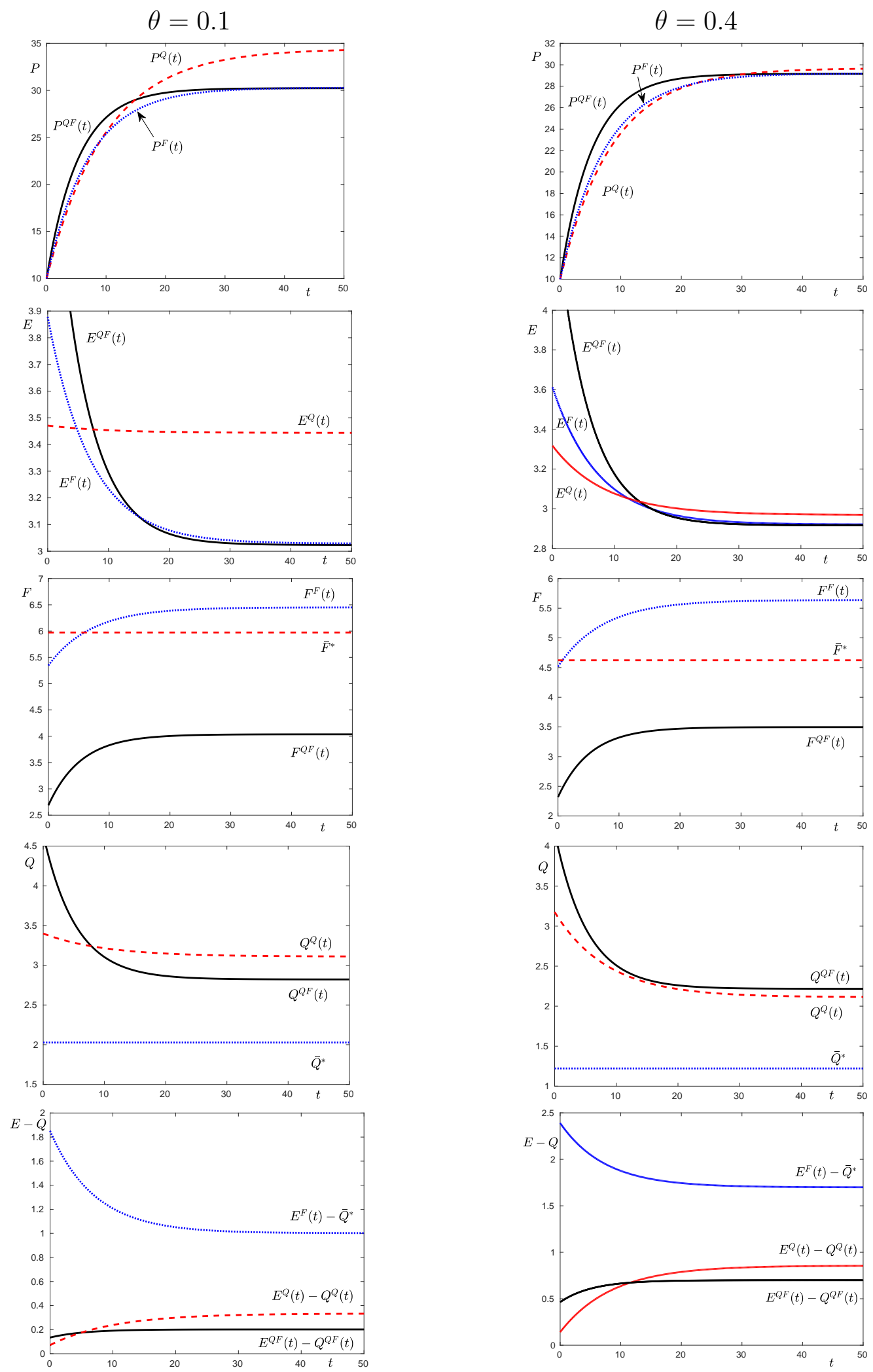

Figure 1: Optimal time-paths for $P, E, F, Q$ and $E-Q$. 
in each scenario for several values for $\theta$, running from $\theta=0$ to $\theta=0.4$. The social welfare is computed by plugging in the integral in (6) the optimal strategies of firm and regulator in each scenario. The analysis is not constrained to the efficient levels of the respective exogenous instruments, but includes a broader set of values $(\bar{F}, \bar{Q})$ (those between 0 and the threshold levels defined in Subsections 4.1 and 4.2). As one would expect, scenario $Q$ becomes more relevant as long as the progressive term of the fine increases. The non-linearity of the fine enables the regulator to induce lower emissions with the emissions limit as the only regulator's decision variable. In the long run, lower emissions will be induced by tightening the emissions limit. Interestingly, a lower standard and lower emissions result regardless of the value of $\bar{F}$. Moreover, the larger $\theta$, the larger social welfare, for any value of $\bar{F}$, as shown in Figure $2 \mathrm{a}^{27}$ In contrast, in scenario $F$, as long as $\theta$ increases, social welfare rises when the constant emission limit, $\bar{Q}$ is tight, but decreases for laxer limit (Figure $2 b$ ).

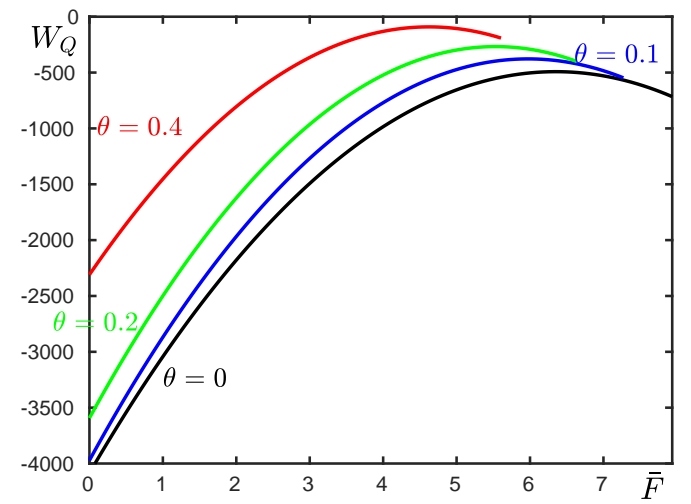

Figure 2a: Scenario $Q$

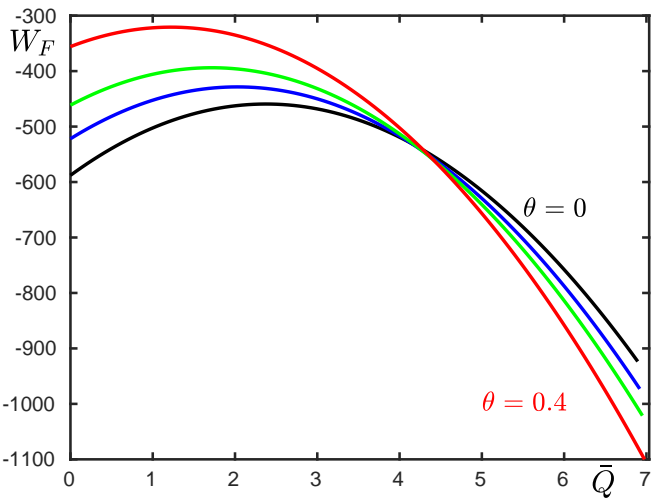

Figure 2b: Scenario $F$

Figure 2: Social welfare in scenarios $Q$ and $F$

Next, we compare social welfare for the two constrained scenarios in Figure 3, for the broader set of values $(\bar{F}, \bar{Q})$ described above, and for three alternative levels of the progressivity of the fine, $\theta=0, \theta=0.1$, and $\theta=0.4$. The charts in the first row refer to the benchmark parameter values in (7). Obviously, social welfare levels at any point are lower than social welfare under scenario $Q F$. All the charts show the level curves for the gap in social welfare between the two scenarios, $W^{F}\left(p_{0}\right)-W^{Q}\left(p_{0}\right)$. The gap is positive in region $F$, while it is negative in region $Q$. Therefore, region $F$ means that scenario $F$ is preferred to scenario $Q$ in social welfare terms; by contrast, region $Q$ means that scenario $Q$ is the preferred scenario.

Note that the comparison between scenarios $Q$ and $F$ is highly dependent on the respective exogenous instruments, which could be set at their corresponding efficient levels $\left(\bar{F}^{*}, \bar{Q}^{*}\right)$

\footnotetext{
${ }^{27}$ When $\theta$ increases, the range of feasible values for $\bar{F}$ narrows (in Figure 2a), while the range of feasible values for $\bar{Q}$ widens slightly (in Figure $2 b$ ).
} 

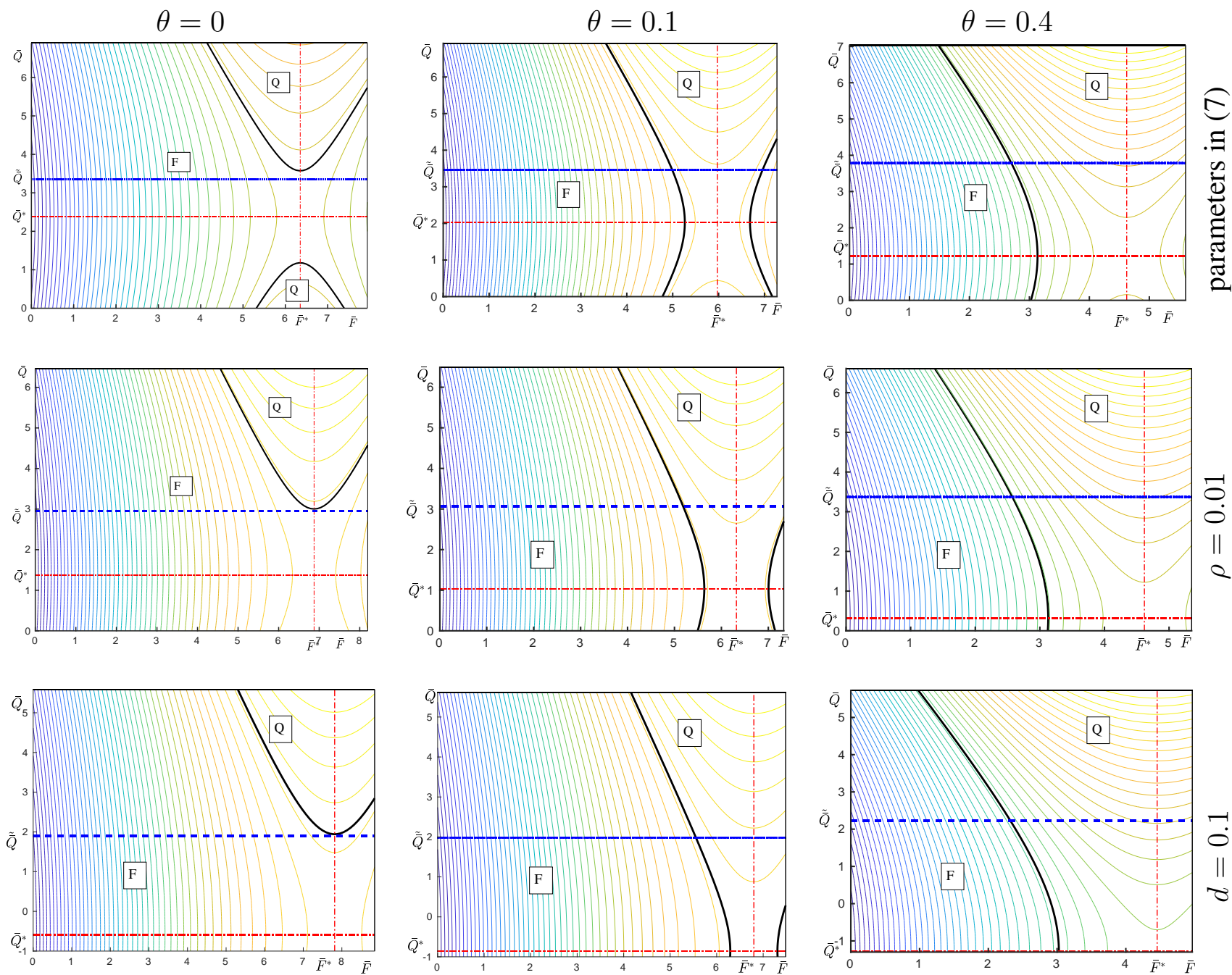

Figure 3: Comparison of social welfares

or not. The point $\left(\bar{F}^{*}, \bar{Q}^{*}\right)$ is highlighted in each figure (and, obviously, it changes when $\theta$ changes) ${ }^{28}$ One possibility is to compare the two scenarios in that point. However, thinking that the government layer responsible for the exogenous instrument could fail to exactly identify the corresponding efficient values, we can consider a neighborhood of $\left(\bar{F}^{*}, \bar{Q}^{*}\right)$ and analyze what scenario is preferred in that neighborhood. Moreover, one would expect the government to be reluctant to fix a constant standard above $\tilde{\bar{Q}}$, which would lead the firm to over-comply, receiving a subsidy (this is identified in the charts by the area above the dashed-blue line). Hence, the region which identifies more likely comparable situations can be centered towards $\left(\bar{F}^{*}, \bar{Q}^{*}\right)$, specifically excluding points with a constant standard above the dashed-blue line $\bar{Q}=\tilde{\bar{Q}}$. While

\footnotetext{
${ }^{28}$ In scenario $Q$, social welfare is maximum at $\bar{F}^{*}$. A higher or a lower $\bar{F}$ (horizontal movements) would reduce social welfare in this scenario, and the gap in social welfare increases. Likewise, in scenario $F$, an emissions standard above or below $\bar{Q}^{*}$ (vertical movements) will reduce social welfare in this scenario, and hence the gap in social welfare.
} 
we focus our analysis on this region, in Figure 3 we plot the wider region for completeness. ${ }^{29}$

When the fine is linear $(\theta=0)$, scenario $F$ provides a higher social welfare than scenario $Q$ at $\left(\bar{F}^{*}, \bar{Q}^{*}\right)$ and the surroundings of this point. This is an expected result, since under a linear fine, the optimal standard does not induce emissions reductions in scenario $Q$. However, Scenario $Q$ can be the preferred scenario if two conditions are simultaneously satisfied. The constant fine $\bar{F}$ must be close to its efficient level $\bar{F}^{*}$ in scenario $Q$, and the constant standard $\bar{Q}$ must be far from its efficient level in scenario $F$. Two regions where scenario $Q$ is preferred arise (see case $\theta=0$ and first row in Figure 3). If the constant emissions limit in scenario $F$ is too tight, emissions and consequently production are too low, while the degree of noncompliance and the associated sanctioning costs are high, implying a small social welfare. ${ }^{30}$ Alternatively, if the constant emission limit is not demanding, the firm has a growing incentive to emit more raising the pollution stock, although emissions remain below the limit, leading to over-compliance, and the associated sanctioning costs. Again, the total effect is a strong decay in social welfare, although this is not a likely situation.

Switching to a non-linear fine $(\theta>0)$ drastically modifies the relative performance of the two scenarios. As shown in the figure $(\theta=0.1$ and $\theta=0.4)$, a positive $\theta$ reverses the roles played by scenarios $Q$ and $F$. With a non-linear fine, the standard gains importance as an enforcement instrument. Scenario $Q$ performs better in terms of social welfare provided that the constant fine $\bar{F}$ is not too far from its efficient level. Conversely, scenario $F$ can only perform better when the error made in this constant fine is very large. As the progressivity of the fine increases, region $Q$ widens, meaning that a greater error can be committed with the constant fine $\bar{F}$, and still this scenario provides higher social welfare than scenario $F$.

Thus, we can conclude the following regarding social welfare:

Claim 2 The prevalence in terms of social welfare moves from scenario $F$ to scenario $Q$, when switching from a linear to a non-linear fine. Moreover, the more progressive the fine, the wider the region at which scenario $Q$ outweighs scenario $F$.

\footnotetext{
${ }^{29}$ In scenario $Q$, we concentrate on values for $\bar{F}$ which do not lead to steady-state pollution stock below its first-best value. Thus, $\bar{F}$ runs between 0 and 7.93, 7.27 and 5.6 for $\theta=0,0.1$ and 0.4 . In fact, this upper bound for the fine is much higher than the marginal damage caused by a rise in emissions when the pollution stock is at its steady-state first-best level which, for the parameters values considered, is given by: $D^{\prime}\left(\tilde{P}^{\mathrm{FB}}\right)=D^{\prime}(20.6)=1.03$. Regarding the emissions limit, there is an upper bound above which the limit would be so lax that firms would be subsidized for over-compliance and would obtain higher benefits than under laissez-faire. We believe these are not likely situations either. Hence, we consider values of $\bar{Q}$ between zero and this upper bound (equal to 6.89, 6.92 and 7.03 for $\theta=0,0.1$ and 0.4 ).

${ }^{30} \mathrm{~A}$ particular case of tight standard in scenario $F$ is $\bar{Q}=0$, which corresponds to the situation where all discharges are penalized (i.e., a tax). The relative performance of scenarios $Q$ and $F$ when $\bar{Q}=0$ is illustrated in the respective horizontal axes of each chart in Figure 3.
} 
As the progressive part of the fine becomes stronger, scenario $Q$ becomes more prevalent also in environmental terms. In Appendix B, we show that the region at which scenario $Q$ leads to lower long-run pollution than scenario $F$ widens with $\theta$.

The robustness of the above results is analyzed with respect to the three parameters which influence the stock pollution problem the most. The second and third rows of Figure 3 show that the results highlighted in Claim 2 remain equally valid after a decrease in the instantaneous discount rate, $\rho$ (from 0.03 to 0.01 ), or an increase in the parameter measuring the damage caused by pollution, $d$ (from 0.05 to 0.1 ). The effect of a decrease in the rate of the regeneration of the pollution stock (or assimilative capacity of the environment), $\delta$, is not presented, but it is rather similar to the effect of $\rho$, as it is usual in this type of dynamic models.

Figure 3 also serves the purpose of analyzing what scenario is better in social welfare terms, when we fix the progressivity of the fine, and we concentrate on the change of the discount rate or environmental damages. For example, starting with the third column in Figure 3, where $\theta=0.4$, we do not see dramatic changes when moving from the benchmark scenario (first row) to the case of a lower discount rate (row 2) or the case of higher environmental damages (row 3). This suggests a strong preference for scenario $Q$ over scenario $F$ as long as the fine is sufficiently progressive in the degree of non-compliance, at least in the surroundings of the respective point $\left(\bar{F}^{*}, \bar{Q}^{*}\right)$. However, the linear case plotted in the first column $(\theta=0)$, shows a larger prevalence of scenario $F$ under a lower discount rate or a higher environmental damage in the cases below the dotted line which separates non-compliance and over-compliance (i.e., the policy relevant cases). The intermediate scenario plotted in the second column $(\theta=0.1)$ shows that the region where scenario $F$ is preferred slightly widens in rows 2 and 3, as compared with the benchmark case plotted in row 1 . All this analysis is summarized as follows:

Claim 3 Ceteris paribus, for relatively small progressivity of the fine for non-compliance, the prevalence of scenario $F$ increases as long as the environmental problem worsens (environmental damages increase or discount rate decreases). These effects diminish as long as the fine is sufficiently progressive in the degree of non-compliance.

Interestingly, the consideration of non-compliance leads to a different result for prices versus quantities as found by Weitzman (1974), and also by Hoel and Karp (2001) for stock pollution. As the environmental problem worsens, the prevalence in the use of prices against the use of standards increases, under a linear or close to linear fine. The reason is that the emissions standard is not very effective in controlling pollution when the fine is not very progressive, and this is particularly important when facing harmful environmental problems. This result, however, diminishes when the fine is progressive enough. 


\section{Constrained solutions II: Players partially ignore the pol- lution stock}

Our dynamic framework allows the regulator to adjust the limit and the fine in accordance with the evolution of the stock of pollution. This section compares the fully dynamic equilibrium presented in Section 3 (Scenario $Q F$ ) with alternative solutions which partially ignore the pollution stock. Firstly, we assume that the regulator is constrained to maintain both the emissions standard and the fine constant while the stock of pollution evolves. ${ }^{31}$ This might be due to practical considerations or implementation issues, since an instrument that does not evolve overtime may be easier to understand by regulated firms. Secondly, we analyze the case of a myopic firm, that decides on emissions considering that the pollution stock does not change overtime. Both cases are analyzed in detail in online Appendix $\mathrm{C}$, here we only present the main results.

\subsection{State-independent equilibrium: Constant strategies}

In the fully dynamic equilibrium the firm was aware that larger emissions, by increasing the pollution stock, resulted in more stringent regulatory policies. That induced a self-restraint effect on the firm, which was captured by a negative marginal valuation of pollution. Now, when the regulator is restricted to constant strategies ( $Q$ and $F$ ), the self-restraint effect does not exist, and the fine per unit must be harsher.

Likewise as in scenario $Q F$, the level of non-compliance is proportional to the fine per unit. And full-compliance occurs under a linear fine specification. Remarkably, and contrary to the solution of scenario $Q F$, these state-independent policies depend on the initial stock of pollution. Thus, if revised at any ulterior time, the regulator will have an incentive to deviate from these strategies. The solution is not time-consistent, and the committed regulatory policies will be more stringent as long as the initial pollution stock is larger.

As expected, in the long run the environment is worse preserved than when the fully dynamic solution is implemented. This result applies regardless of whether the environmental problem is already initially serious or not very harmful. Because the state-independent equilibrium is restricted to constant strategies, this solution must be suboptimal in social welfare terms with respect to the fully dynamic solution. This difference is more pronounced the higher the initial pollution stock is.

We have carried out simulations for different values of $\theta$ and obtained qualitatively similar results. The main effects of a higher $\theta$ are lower emissions and pollution stock. Because a

\footnotetext{
${ }^{31}$ The cases where the regulator is compelled to maintain one of the enforcement instruments constant and optimally choose the other have already been presented in scenario $Q$ and $F$, where we computed the efficient value of the constant instrument.
} 
higher $\theta$ gives prominence to the standard, this instrument becomes slightly more demanding, while the fine per unit becomes laxer.

\subsection{Myopic firm}

We now consider that a myopic agent takes his/ her decision as if the pollution stock was constant. We focus on the case with a myopic firm and a farsighted regulator. ${ }^{32}$ Being myopic, the firm does not acknowledge how emissions influence the pollution stock and consequently the regulatory policy. Again the firm lacks self-restraint, and the level of non-compliance is proportional to the fine per unit. However, contrary to the state-independent equilibrium, the myopic equilibrium is subgame perfect.

The myopic case is characterized by a more punishing fine per unit, while at the same time the standard is less demanding. All in all, the emissions, and consequently the pollution stock grow higher when the firm behaves myopically. As one would expect, the myopic behaviour of the firm reduces social welfare, and the reduction is more pronounced with a higher initial pollution stock.

\section{Conclusions}

In this paper, we have studied the combination of optimal pollution standards and fines for noncompliance in problems where pollution accumulates overtime, and resulting environmental damages depend on the pollution stock. We have considered a differential game played à la Stackelberg, with one-leading regulator and one follower-firm. Although several studies have analyzed alternative forms of regulation in stock pollution problems (such as Biglaiser et al., 1995, Hoel and Karp, 2001, Xabadia et al., 2008, or Karp and Zhang, 2016), however, none of them considers the possibility that firms exceed the regulations.

Our study is the first to incorporate non-compliance in stock pollution problems. Our focus is clearly different from the literature, since we concentrate on the effects of non-compliance (particularly, on the importance of each regulatory instrument in the marginal fine, and the corresponding effect on firms' emissions), while, at the same time, we acknowledge symmetric information between the regulator and the firm. This different focus results in a different conclusion for the prevalence of prices versus quantities. As a result, the relative importance of

\footnotetext{
${ }^{32}$ The case in which both agents are myopic is not interesting, because then the regulator would choose a zero penalty and an emission limit identical to the laissez-faire emissions. The alternative scenario with a farsighted firm that acknowledges the effect of its decisions on the pollution stock and a myopic regulator does not seem realistic.
} 
non-compliance and asymmetric information in the problem at hand may then condition the preference for one type of regulation versus the other.

We have analyzed a flexible benchmark scenario where both the standard and the enforcement mechanism are policy variables that evolve overtime (scenario $Q F$ ). Then, we have considered different restrictive scenarios. On the one hand, we have assumed that only the enforcement instrument or only the standard are policy controls (scenarios $F$ and $Q$, respectively), while the alternative instrument is exogenous. On the other hand, we have considered that agents (the regulator or the firm) partially ignore the pollution stock, leading to policies that can be, in principle, more easily implemented, but to the expense that they are not time consistent.

A key element in our analysis is the structure of the fine, specifically, how progressive the fine is with respect to the degree of non-compliance. In the particular case where the fine is linear, the marginal fine is constant, and the standard has no effect on firm's emissions. It turns out that controlling through fines is better in social welfare terms than controlling through standards, specially when the environmental problem is sufficiently harmful (everything else constant, when marginal damages increase, or the discount factor or the degree of assimilative capacity decrease).

As long as the fine becomes progressive and as the progressivity increases, the standard starts to influence emissions (its effect on emissions increases with respect to the corresponding effect of the -linear part of- the fine). This means that the prevalence of controlling through standards increases with the progressivity of the fine. In the terminology of our paper, as the non-linearity of the fine strengthens, scenario $Q$ more closely approaches the benchmark scenario $Q F$, and greatly overcomes scenario $F$ in social welfare terms. This effect seems to be strong, since it persists regardless of the specific parameters describing the nature of the environmental problem.

Our main message is that setting standards dependent on the pollution stock seems to be preferred if fines are sufficiently non-linear. However, setting fines dependent on the pollution stock seems to be preferred if fines are linear or almost linear, and specially in cases where the environmental problem is particularly harmful. Interestingly, our results are contrary to Hoel and Karp (2001), among others. In a context of full compliance and asymmetric information between the regulator and the firms, they show the particular preference for quantities in cases with larger marginal damages, or lower discount factor or degree of assimilative capacity, also in accordance with Weitzman (1974) in the static context. Our analysis, however, suggests the preference for price regulation if non-compliance is an issue (and asymmetric information is not). All this seems to suggest that the relative importance of non-compliance and asymmetric information in the problem at hand may then condition the preference for prices versus quantities. 
Mainly due to tractability considerations, our model contains several, to some extent, controversial assumptions, and it may be surely worth to explore if our analysis extends to more realistic situations. For example, linked to the assumption of symmetric information, our analysis abstracts from the monitoring of emissions, but in reality, monitoring is costly, and oftentimes imperfect and subject to errors. For example, the analysis in Harrington (1988) and subsequent papers on optimal dynamic enforcement can be worth to incorporate in our setting. Second, our model deals with the case of a representative firm, which allows us to avoid noise coming from the output market, relation to rivals, and/or regulatory issues related to the presence of heterogeneous polluters. There is an extensive literature on dynamic regulation of diffuse wastewater sources (see, for example, Goetz and Xabadia, 2015, for an extensive review) that may be interesting to combine with our setting, since the enforcement aspect is ignored in all these studies. Finally, our analysis assumes that only one regulatory body is responsible for setting the pollution standard and/or the enforcement instrument, while in most cases, different layers of the Government are responsible for the control of each instrument. Interesting coordination and hierarchy issues can arise in this case, as in Arguedas et al. (2017b), among others. We think all these issues deserve further investigation.

\section{References}

[1] Arguedas, C. (2008), To Comply or Not to Comply? Pollution Standard Setting under Costly Monitoring and Sanctioning. Environmental and Resource Economics 41, 155168.

[2] Arguedas, C. (2013), Pollution Investment, Technology Choice and Fines for NonCompliance. Journal of Regulatory Economics 44, 156-176.

[3] Arguedas, C., F. Cabo, and G. Martín-Herrán (2017a), Optimal Pollution Standards and Non-Compliance in a Dynamic Framework. Environmental and Resource Economics 68, 537-567.

[4] Arguedas, C., D. Earnhart, and S. Rousseau (2017b), Non-Uniform Implementation of Uniform Standards. Journal of Regulatory Economics 51, 159-183.

[5] Beavis, B., and I.M. Dobb (1986), The Dynamics of Optimal Environmental Regulation. Journal of Economic Dynamics and Control 10, 415-423.

[6] Benford, F.A. (1998), On the Dynamics of the Regulation of Pollution: Incentive Compatible Regulation of a Persistant Pollutant. Journal of Environmental Economics and Management 36, 1-25. 
[7] Biglaiser, G., Horowitz, J.K. and J. Quiggin (1995), Dynamic Pollution Regulation. Journal of Regulatory Economics 8, 33-44.

[8] Billiet, C.A., T. Blondiau, and S. Rouseau (2014), Punishing Environmental Crimes: An Empirical Study from Lower Courts to the Court of Appeal. Regulation and Governance $8,472-496$.

[9] Chen, C.C., and Y.B. Lai (2012), Does a Stricter Enforcement Policy Protect the Environment? A Political Economy Perspective. Resource and Energy Economics 34, 431-441.

[10] Conrad, M. (1992), Stopping Rules and the Control of Stock Pollutants. Natural Resource Modelling 6, 315-327.

[11] Downing, P.B., and W.D. Watson (1974), The Economics of Enforcing Air Pollution Controls. Journal of Environmental Economics and Management 1, 219-236.

[12] Earnhart, D. (1997), Enforcement of Environmental Protection Laws Under Communism and Democracy. Journal of Law and Economics 40, 377-402.

[13] Falk, I., and R. Mendelsohn (1993), The Economics of Controlling Stock Pollutants: An Efficient Strategy for Greenhouse Gases. Journal of Environmental Economics and Management 25, 76-88.

[14] Friesen, L. (2003), Targeting Enforcement to Improve Compliance with Environmental Regulations. Journal of Environmental Economics and Management 46, 72-85.

[15] Goetz, R., and A. Xabadia (2015), Externalities and Water Quality. In: R. Howitt, J. Roumasset, and K. Burnett (eds) Handbook of Water Economics and Institutions. Routledge, Taylor \& Francis Group. New York.

[16] Harford, J.D. (1978), Firm Behavior Under Imperfectly Enforceable Pollution Standards and Taxes. Journal of Environmental Economics and Management 5, 26-43.

[17] Harford J.D., and W. Harrington (1991), A Reconsideration of Enforcement Leverage When Penalties Are Restricted. Journal of Public Economics 45, 391-395.

[18] Harrington, W. (1988), Enforcement Leverage When Penalties Are Restricted. Journal of Public Economics 37, 29-53.

[19] Hartl, R.F. (1992), Optimal Acquisition of Pollution Control Equipment Under Uncertainty. Management Science 38, 609-622. 
[20] Haurie, A., J.B. Krawczyk, and G. Zaccour (2012), Games and Dynamic Games. World Scientific, Singapore.

[21] Hoel, M. and L. Karp (2001), Taxes and Quotas for a Stock Pollutant with Multiplicative Uncertainty. Journal of Public Economics 82(1), 91-114.

[22] Jones, C.A., and S. Scotchmer (1990), The Social Cost of Uniform Regulatory Standards in a Hierarchical Government. Journal of Environmental Economics and Management 19, 61-72.

[23] Jørgensen, S., G. Martín-Herrán and G. Zaccour (2010), Dynamic Games in the Economics and Management of Pollution. Environmental Modeling and Assessment 15 (6), 433-467.

[24] Karp, L. and J. Zhang (2016), Taxes Versus Quantities for a Stock Pollutant with Endogenous Abatement Costs and Asymmetric Information. In: Chichilnisky G., Rezai A. (eds) The Economics of the Global Environment. Studies in Economic Theory, vol 29, pp. 493-533. Springer, Cham.

[25] Keeler, A. (1995), Regulatory Objectives and Enforcement Behavior. Environmental and Resource Economics 6, 73-85.

[26] Kleit, A.N., M.A. Pierce, and R.C. Hill (1998), Environmental Protection, Agency Motivations and Rent Extraction: The Regulation of Water Pollution in Louisiana. Journal of Regulatory Economics 13, 121-137.

[27] Lefebvre, O., and R. Moletta (2006), Treatment of Organic Pollution in Industrial Saline Wastewater: A literature Review. Water Research 40, 3671-3682.

[28] Oljaca N., A.G. Keeler, and J. Dorfman (1998), Penalty Functions for Environmental Violations: Evidence from Water Quality Enforcement. Journal of Regulatory Economics 14, 255-264.

[29] Polinsky, A.M., and S. Shavell (1992), Enforcement Costs and the Optimal Magnitude and Probability of Fines. Journal of Law and Economics 35, 133-148.

[30] Raymond, M. (1999), Enforcement Leverage When Penalties Are Restricted: A Reconsideration Under Asymmetric Information. Journal of Public Economics 73, 289-295.

[31] Rousseau, S. (2007), Economic Empirical Analysis of Sanctions for Environmental Violations: a Literature Overview. Working Paper Series 2007-03, Energy, Transport and Environment, KU Leuven. 
[32] Stranlund, J.K. (2007), The Regulatory Choice of Non-Compliance in Emission Trading Programs. Environmental and Resource Economics 38, 99-117.

[33] Xabadia, A., Goetz, R.U. and D. Zilberman (2008), The Gains from Differentiated Policies to Control Stock Pollution when Producers Are Heterogeneous. American Journal of Agricultural Economics 90(4), 1059-1073.

[34] Weitzman, M. (1974), Prices versus Quantities. Review of Economic Studies 41(4), 477491.

[35] Zhang, X.B., and J. Xu (2016), A Dynamic Enforcement Strategy to Improve Compliance with Environmental Regulations. Environment for Development, Discussion Paper Series 16-17. 


\section{Online Appendix}

\section{Appendix A: Stagewise Feedback Stackelberg Equilibria}

In a first step, the firm, acting as the follower, computes its best-reaction function by solving the Hamilton-Jacobi-Bellman equation associated with problem (4):

$$
\rho V^{s}(P)=\max _{E}\left\{Y(E)-G(E, Q, F)+\left(V^{s}\right)^{\prime}(P)(E-\delta P)\right\}
$$

with $V^{s}(P)$ the firm's value function and $s=\{Q, F, Q F\}$. Taking into account (1) and (3) the first-order condition leads to:

$$
\widehat{E}^{s}(P ; Q, F)=\frac{\sigma+\theta Q-F+\left(V^{s}\right)^{\prime}(P)}{1+\theta} .
$$

In a second step, the regulator who takes the role of the leader, knowing the firm's best-reaction function, has to determine the optimal $Q, F$, or both in order to solve problem (6). Then, the Hamilton-Jacobi-Bellman equation for the regulator reads:

$\rho W^{s}(P)=\max _{Q, F}\left\{Y\left(\widehat{E}^{s}(P ; Q, F)\right)-D(P)-H\left(\widehat{E}^{s}(P ; Q, F), Q, F\right)+\left(W^{s}\right)^{\prime}(P)\left(\widehat{E}^{s}(P ; Q, F)-\delta P\right)\right\}$,

with $W^{s}(P)$ the regulator's value function. Taking into account (1) and (5), the optimality conditions for this problem can be written as:

$$
\begin{aligned}
& (\sigma-Q)\left(\beta+\theta^{2}\right)-F(\beta-\theta)+(\beta-\theta)\left(V^{s}\right)^{\prime}(P)+\theta(1+\theta)\left(W^{s}\right)^{\prime}(P)=0 \\
& (\sigma-Q)(\beta-\theta)-F\left(1+\beta+(1+\theta)^{2} \xi\right)+(1+\beta)\left(V^{s}\right)^{\prime}(P)-(1+\theta)\left(W^{s}\right)^{\prime}(P)=0
\end{aligned}
$$

The optimal strategy in scenario $Q$ (resp. $F$ ) is computed taking into account the first (resp. second) equation. The optimal strategies in scenario $Q F$ are computed by considering both equations.

Given the linear-quadratic structure of the game, quadratic value functions are conjectured for the firm, $V^{s}(P)=v_{2}^{s} P^{2} / 2+v_{1}^{s} P+v_{0}^{s}$, and the regulator, $W^{s}(P)=w_{2}^{s} P^{2} / 2+w_{1}^{s} P+w_{0}^{s}$. Substituting, in each scenario, the corresponding first-order conditions in the Hamilton-JacobiBellman equations, taking into account the conjectured value functions and equating coefficients of the same order, a system of six Riccati equations is obtained for the unknown coefficients $v_{i}^{s}, w_{i}^{s}, i \in\{0,1,2\}$. In general for this non-linear coupled system closed-form solutions are not easily found. Thus, we rely on numerical simulations computed for the parameters values in (7).

Four possible pairs of solutions for coefficients $v_{2}^{s}$ and $w_{2}^{s}$ arise from the Riccati equations. In the three scenarios two of these solutions are complex numbers. In scenarios $Q F$ and $Q$ a third solution can be removed as it would imply a non-asymptotically stable steady-state. In 
scenario $F$, focusing only on interior real solutions avoiding the analysis of corner solutions, a third solution can also be removed. Therefore, a unique solution for coefficients $v_{i}^{s}, w_{i}^{s}$ is identified in each of the three scenarios.

The optimal time-path for the pollution stock in each scenario reads:

$$
P^{s}(t)=\left(p_{0}-\tilde{P}^{s}\right) e^{\phi^{s} t}+\tilde{P}^{s}
$$

where $\tilde{P}^{s}$ and $\phi^{s}$ are the steady state of the pollution stock and the speed of convergence given by:

$$
\begin{aligned}
& \tilde{P}^{Q F}=\frac{\sigma\left(\beta(1+\xi)+\theta^{2} \xi\right)+\left(\beta+\theta^{2} \xi\right) w_{1}^{Q F}+\beta \xi v_{1}^{Q F}}{\delta\left(\beta(1+\xi)+\theta^{2} \xi\right)-\left(\beta+\theta^{2} \xi\right) w_{2}^{Q F}-\beta \xi v_{2}^{Q F}}, \quad \phi^{Q F}=\frac{\left(\beta+\theta^{2} \xi\right) w_{2}^{Q F}+\beta \xi v_{2}^{Q F}}{\beta(1+\xi)+\theta^{2} \xi}-\delta, \\
& \tilde{P}^{Q}=\frac{\sigma\left(\beta+\theta^{2}\right)-\beta \bar{F}+\theta^{2} w_{1}^{Q}+\beta v_{1}^{Q}}{\delta\left(\beta+\theta^{2}\right)-\theta^{2} w_{2}^{Q}-\beta v_{2}^{Q}}, \quad \phi^{Q}=\frac{\theta^{2} w_{2}^{Q}+\beta v_{2}^{Q}}{\beta+\theta^{2}}-\delta, \\
& \tilde{P}^{F}=\frac{\sigma(1+(1+\theta) \xi)+((1+\theta) \xi \theta+\beta) \bar{Q}+w_{1}^{F}+(1+\theta) \xi v_{1}^{F}}{\delta\left(1+\beta+(1+\theta)^{2} \xi\right)-w_{2}^{F}-(1+\theta) \xi v_{2}^{F}}, \quad \phi^{F}=\frac{w_{2}^{F}+(1+\theta) \xi v_{2}^{F}}{1+\beta+(1+\theta)^{2} \xi}-\delta,
\end{aligned}
$$

\section{Laissez-Faire}

Under laissez-faire the firm chooses emissions ignoring the environmental problem it causes. Thus, the firm maximizes production at each time and, therefore, ${ }^{33} E^{\mathrm{U}}=\sigma$. At this constant level of emissions, the optimal pollution stock evolves according to $P^{\mathrm{U}}(t)=\left(p_{0}-\tilde{P}^{\mathrm{U}}\right) e^{-\delta t}+\tilde{P}^{\mathrm{U}}$, with the steady-state pollution stock given by $\tilde{P}^{\mathrm{u}}=\sigma / \delta$. The value functions (aggregated optimal discounted welfares) for the firm, $V^{\mathrm{U}}$, and for the society, $W^{\mathrm{U}}$, can be expressed respectively as:

$$
\begin{aligned}
& V^{\mathrm{U}}\left(p_{0}\right)=\int_{0}^{\infty} Y(\sigma) e^{-\delta t} d t=\frac{\sigma^{2}}{2 \rho}, \\
& W^{\mathrm{U}}\left(p_{0}\right)=\int_{0}^{\infty}\left[Y(\sigma)-D\left(P^{\mathrm{U}}(t)\right)\right] e^{-\delta t} d t=w_{2}^{\mathrm{U}} \frac{p_{0}^{2}}{2}+w_{1}^{\mathrm{U}} p_{0}+w_{0}^{\mathrm{U}}, \\
& w_{2}^{\mathrm{U}}=-\frac{d}{\rho+2 \delta}, \quad w_{1}^{\mathrm{U}}=-\frac{d \sigma}{(\rho+2 \delta)(\rho+\delta)}, \quad w_{0}^{\mathrm{U}}=\frac{\sigma^{2}}{2 \rho}-\frac{d \sigma^{2}}{\rho(\rho+2 \delta)(\rho+\delta)} .
\end{aligned}
$$

\section{First-Best solution}

The first-best or social optimum represents the case of either a maximizing firm which fully internalizes the environmental damage, or equivalently, that of a regulator who directly fixes

\footnotetext{
${ }^{33}$ Superscript $U$ stands for unregulated scenario.
} 
emission with no need to enforce them. The optimization problem can be written as:

$$
\begin{gathered}
\max _{E} \int_{0}^{\infty}[Y(E)-D(P)] e^{-\rho t} d t \\
\text { s.t.: } \dot{P}=E-\delta P, \quad P(0)=p_{0} .
\end{gathered}
$$

Optimal emissions are fixed at the level at which the instantaneous marginal benefit of emissions through output, $\sigma-E$, equates the ongoing marginal damage of the associated increment in the stock of pollution for society ${ }^{34},-\left(W^{\mathrm{FB}}\right)^{\prime}(P)$. Thus, emissions are given by the feedback rule $E^{\mathrm{FB}}(P)=\sigma+\left(W^{\mathrm{FB}}\right)^{\prime}(P)$. The value function is conjectured quadratic, as follows: $W^{\mathrm{FB}}(P)=$ $w_{2}^{\mathrm{FB}} P^{2} / 2+w_{1}^{\mathrm{FB}} P+w_{0}^{\mathrm{FB}}$. The unique convergent first-best solution implies an optimal time-path for the pollution stock given by:

$$
\begin{aligned}
& P^{\mathrm{FB}}(t)=\left(p_{0}-\tilde{P}^{\mathrm{FB}}\right) e^{\phi^{\mathrm{FB}} t}+\tilde{P}^{\mathrm{FB}}, \\
& \tilde{P}^{\mathrm{FB}}=\frac{(\rho+\delta) \sigma}{d+\delta(\rho+\delta)}, \quad \phi^{\mathrm{FB}}=\frac{\rho-\sqrt{\Theta^{\mathrm{FB}}}}{2}<0, \quad \Theta^{\mathrm{FB}}=(\rho+2 \delta)^{2}+4 d,
\end{aligned}
$$

where $\tilde{P}^{\mathrm{FB}}$ is the steady-state pollution stock in the first best. It is immediately obvious that $\tilde{P}^{\mathrm{FB}}<\tilde{P}^{\mathrm{U}}$ and $\phi^{\mathrm{FB}}<-\delta<0$. Therefore, the first-best pollution stock converges towards a long-run value below the stock under laissez-faire, and it converges faster. The coefficients of the value function are given by:

$$
w_{2}^{\mathrm{FB}}=\frac{\rho+2 \delta-\sqrt{\Theta^{\mathrm{FB}}}}{2}<0, \quad w_{1}^{\mathrm{FB}}=\sigma \frac{\rho+2 \delta-\sqrt{\Theta^{\mathrm{FB}}}}{\rho+\sqrt{\Theta^{\mathrm{FB}}}}<0, \quad w_{0}^{\mathrm{FB}}=\frac{2 \sigma^{2}(\rho+\delta)^{2}}{\rho\left(\rho+\sqrt{\Theta^{\mathrm{FB}}}\right)^{2}}>0 .
$$

It can be proved that $w_{2}^{\mathrm{FB}}>w_{2}^{\mathrm{U}}, w_{1}^{\mathrm{FB}}>w_{1}^{\mathrm{U}}$ and $w_{0}^{\mathrm{FB}}>w_{0}^{\mathrm{U}}$. Therefore, it follows that $W^{\mathrm{FB}}(P)>$ $W^{\mathrm{U}}(P)$ for any $P \geq 0$. Moreover the marginal decay in the social welfare with pollution is stronger under laissez-faire, $0>\left(W^{\mathrm{FB}}\right)^{\prime}(P)>\left(W^{\mathrm{U}}\right)^{\prime}(P)$ for any $P \geq 0$.

\section{Appendix B: Comparison of long-run pollution stocks}

In this appendix, we compare the two single-instrument scenarios (and both to the benchmark scenario $Q F$ ). In Figure 4, we compare the steady-state values of the pollution stock for these scenarios and for different values of the constrained instruments, $(\bar{F}, \bar{Q})$. This figure displays in the $\bar{F} \bar{Q}$-plane the regions that result in the lowest steady-state value of the pollution stock for $\theta=0.1$ (Figure $4 \mathrm{a}$ ), and for $\theta=0.4$ (Figure $4 \mathrm{~b}$ ).

First note that the steady-state value of the pollution stock under scenario $Q F$ is independent of the specific constraints used in the other two scenarios, $\bar{F}$ or $\bar{Q}$, and hence, it is constant

\footnotetext{
${ }^{34}$ Superscript $F B$ stands for the first-best scenario.
} 


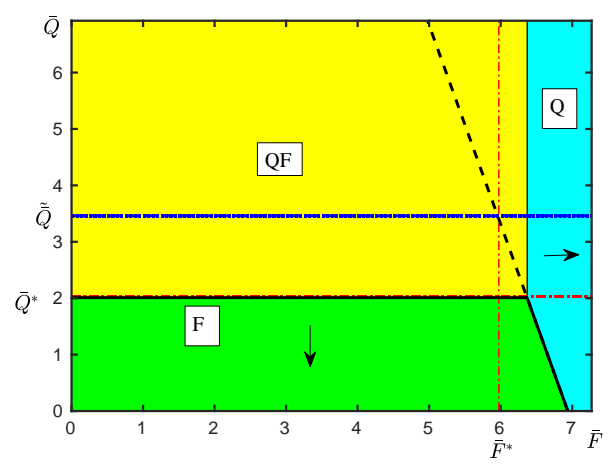

Figure 4a: $\theta=0.1$

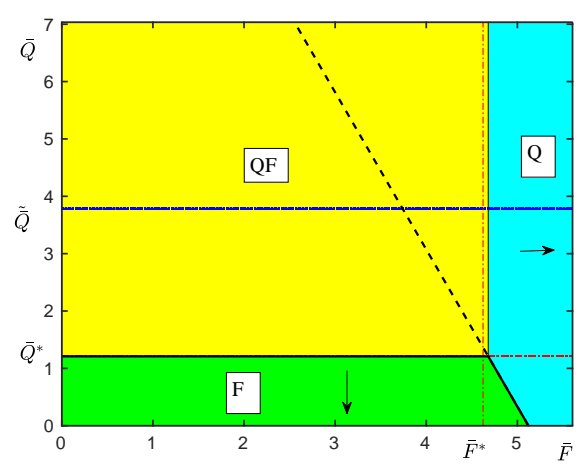

Figure 4b: $\theta=0.4$

Figure 4: Comparison of long-run pollution stocks in the three scenarios

across the $\bar{F} \bar{Q}$-plane. Under scenario $F, \bar{Q}$ is given, and the steady-state pollution stock is constant for a given $\bar{Q}$ (moving horizontally); while it decreases as long as $\bar{Q}$ becomes tighter, (moving downwards). Conversely, under scenario $Q, \bar{F}$ is given, and the steady-state pollution stock in this scenario is constant for given $\bar{F}$ (moving vertically); while it decreases as long as $\bar{F}$ becomes larger, (moving horizontally to the right). In this sense, the arrows in the figure represent decays in the steady-state pollution stock of the best preserving scenario in this region.

To analyze the graphs in Figure 4, we start at point $\left(\bar{F}^{*}, \bar{Q}^{*}\right)$ around which it is more likely to identify real live situations. Up-left of this point, where either the exogenously constant fine in scenario $Q$ or the constant limit in scenario $F$ are lax, scenario $Q F$ is preferred in environmental terms. Outside this region, the scenario with a tougher restricted instrument tends to lead to a lower long-run pollution. However, which scenario leads to lower pollution depends on $\theta$. The main conclusions is that the greater the progressivity of the fine for non-compliance, $\theta$, the wider is the region at which scenario $Q$ outweighs scenario $F$ in environmental terms.

\section{Appendix C: Constrained solutions II: Players partially ignore the pollu- tion stock}

\section{State-independent equilibrium: Constant strategies}

We now consider that the Stackelberg dynamic game in (4) and (6) is solved restricted to constant strategies $(Q$ and $F)$ for the regulator. Because the fine per unit and the standard are independent of the pollution stock, the firm is a static player whose reaction function is given by: ${ }^{35}$

$$
\hat{E}^{\mathrm{C}}(Q, F)=\frac{\sigma+\theta Q-F}{1+\theta} .
$$

\footnotetext{
${ }^{35}$ Superscript $C$ stands for constant strategies.
} 
This expression differs from the reaction function in the fully dynamic equilibrium, given by expression (8). In that case the firm was aware that larger emissions, by increasing the pollution stock, resulted in more stringent regulatory policies. That induced a self-restraint effect on the firm, which was captured by a negative marginal valuation of pollution. With state-independent strategies, the self-restraint effect does not exist, and the fine per unit must be harsher.

Plugging $\hat{E}^{\mathrm{C}}(Q, F)$ in (15) into (2), the pollution stock time-path can be computed as a function of the constants $Q$ and $F$. From the temporal evolution of the pollution stock, the social welfare (given by the integral in (6)) can be computed as a function of the unknown constants $Q$ and $F$. It is now straightforward to compute their optimal constant values:

$$
\begin{aligned}
& Q^{\mathrm{C}}=\sigma-\frac{d(\beta+\theta(1+\theta) \xi)\left(\rho p_{0}+2 \sigma\right)}{2 d\left(\beta+\theta^{2} \xi\right)+\left[\beta+\left(\theta^{2}+\beta\right) \xi\right](\delta+\rho)(2 \delta+\rho)}, \\
& F^{\mathrm{C}}=\frac{\beta d\left(\rho p_{0}+2 \sigma\right)}{2 d\left(\beta+\theta^{2} \xi\right)+\left[\beta+\left(\theta^{2}+\beta\right) \xi\right](\delta+\rho)(2 \delta+\rho)} .
\end{aligned}
$$

From these expressions and the reaction function of the firm, it immediately follows that the level of non-compliance is given by $E^{\mathrm{C}}-Q^{\mathrm{C}}=\theta \xi F^{\mathrm{c}} / \beta$. Likewise as in scenario $Q F$ (the fully dynamic equilibrium), the equilibrium is characterized by full-compliance under a linear fine specification. Remarkably, and contrary to the solution of scenario $Q F$, this state-independent policies depend on the initial stock of pollution. Thus, if revised at any ulterior time, the regulator will have an incentive to deviate from these strategies. The time-inconsistency of the solution results by construction, since this solution concept restricts to constant strategies which requires the regulator's commitment. The committed regulatory policies will be more stringent the larger the initial pollution stock.

To compare scenario $Q F$ and the state-independent equilibrium, in Figure 5 we first assume a small initial pollution stock $\left(p_{0}=10\right)$. The analysis is carried out for the parameters values in (7), and further assuming $\theta=0.1$. In both scenarios, the pollution stock increases. As the environmental problem aggravates, in the flexible scenario $Q F$ the regulator has the capacity to strengthen the fine per unit and the emissions standard. The optimal fine in the flexible scenario never reaches the constant fine per unit in the state-independent equilibrium (which lacks the self-restraint effect), while the flexible standard crosses the constant standard from above. Emissions are initially larger but, from a given moment on, they remain below the emissions in the state-independent scenario. In consequence, although initially a state-independent equilibrium is associated with a less polluted environment, it will lead to a greater steady-state pollution stock in the long run. Finally, the level of non-compliance in the fully dynamic scenario grows with time, although it never reaches its level in the state-independent case.

Conversely, starting from an initially large pollution stock $\left(p_{0}=45\right)$, it will decrease overtime. In the fully dynamic case, the fine per unit already starts below its constant value in the 


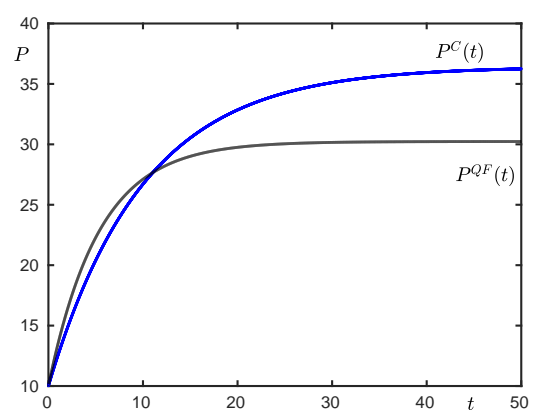

Figure 5a: Pollution stock

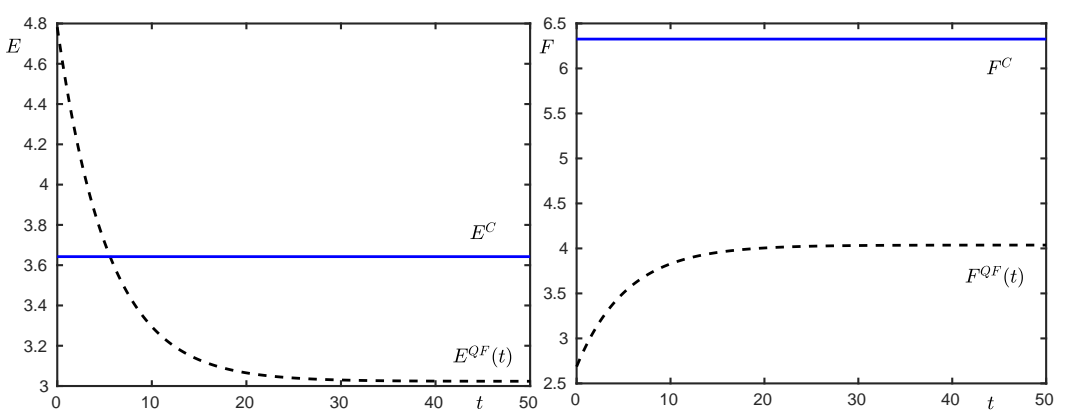

Figure 5b: Emissions

Figure 5c: Fine per unit

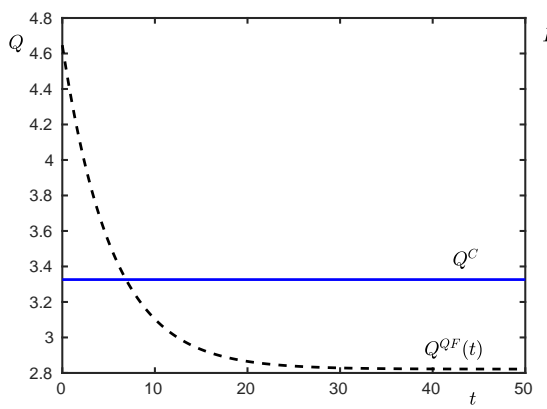

Figure 5d: Emissions standard

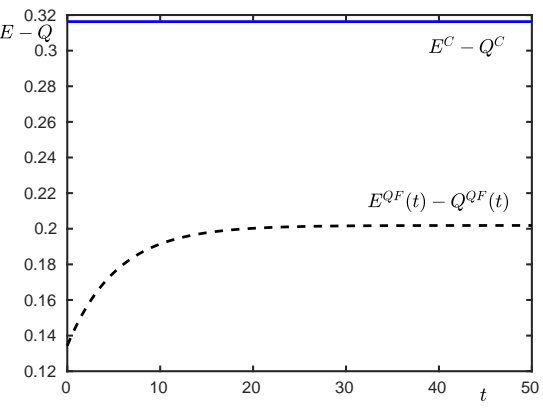

Figure 5e: Non-compliance

Figure 5: Optimal time-paths: fully dynamic versus state-independent equilibrium.

state-independent equilibrium and decreases even further with time. The emissions and the standard increase with time although they never reach their constant values. Therefore the pollution stock decreases more sharply and to a lower steady state than in the state-independent equilibrium.

In consequence, as expected, the environment is better preserved in the long run when the fully dynamic solution is implemented. This result applies regardless of whether the environmental problem is already initially serious or not very harmful. Because the state-independent equilibrium is restricted to constant strategies, this solution must be suboptimal (in terms of social welfare) with respect to the fully dynamic solution. This difference is more pronounced the higher is the initial pollution stock.

We have carried out simulations for values of $\theta$ different from 0.1 and obtained qualitatively similar results. The main effect of a higher $\theta$ is lower emissions and pollution stock. Because a higher $\theta$ gives prominence to the standard, this instrument becomes slightly more demanding, while the fine per unit becomes laxer. 


\section{Myopic firm}

We now consider that a myopic agent takes his/ her decision as if the pollution stock was constant. We focus on the case with a myopic firm and a farsighted regulator. ${ }^{36}$ Being myopic, the firm does not acknowledge how emissions influence the pollution stock and consequently the regulatory policy. The firm again lacks self-restraint and its reaction function is thus given by the expression in (15). Introducing this reaction function and solving the dynamic problem for the regulator in (6), one gets the optimal feedback strategies for the standard and the fine per unit as: $:^{37}$

$$
Q^{\mathrm{M}}(P)=\sigma+\frac{(\beta+\theta(1+\theta) \xi)\left(W^{\mathrm{M}}\right)^{\prime}(P)}{\beta+\left(\theta^{2}+\beta\right) \xi}, \quad F^{\mathrm{M}}(P)=-\frac{\beta\left(W^{\mathrm{M}}\right)^{\prime}(P)}{\beta+\left(\theta^{2}+\beta\right) \xi},
$$

where $W^{\mathrm{M}}(P)$ represents the regulator's value function. The equilibrium characterized by these strategies is subgame perfect. In this scenario, since the regulator determines both instruments, again the level of non-compliance is proportional to the fine per unit: $E^{\mathrm{M}}-Q^{\mathrm{M}}=\theta \xi F^{\mathrm{M}} / \beta$.

A myopic firm attaches a zero value to pollution, contrary to a farsighted firm which negatively values pollution. This helps compare expressions in (9) and (10) with (16). As Figure 6 shows, the myopic case is characterized by a more punishing fine per unit, while at the same time the standard is less demanding. All in all, the emissions, and consequently the pollution stock grow higher when the firm behaves myopically. The regulator cannot push the myopic firm to reach its farsighted emissions, as this will require an excessively large fine per unit, which will disproportionately rise the regulator's costs. Because the emissions and consequently the level of non-compliance would be too large in the myopic case, the regulator relax the standard to smooth sanctioning costs. As one would expect, the myopic behaviour of the firm reduces social welfare, and the reduction is more pronounced, the higher is the initial pollution stock.

\footnotetext{
${ }^{36}$ The case in which both agents are myopic is not interesting, because then the regulator would choose a zero penalty and an emission limit identical to the laissez-faire emissions. The alternative scenario with a farsighted firm that acknowledges the effect of its decisions on the pollution stock and a myopic regulator does not seem realistic.

${ }^{37}$ Superscript $M$ stands for myopic firm.
} 


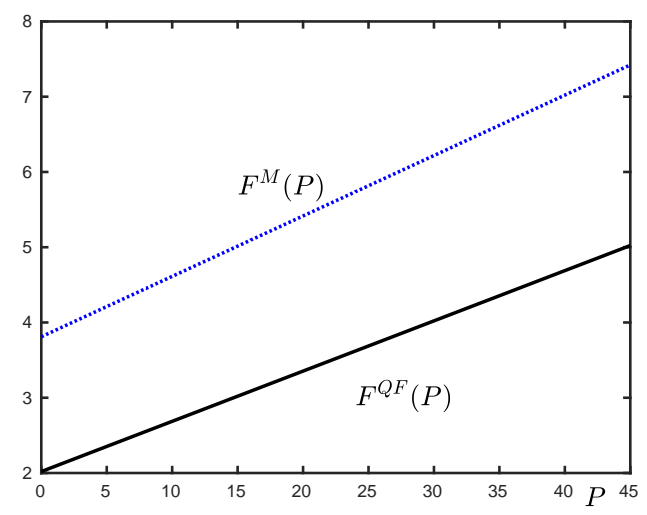

Figure 6a: Fine per unit

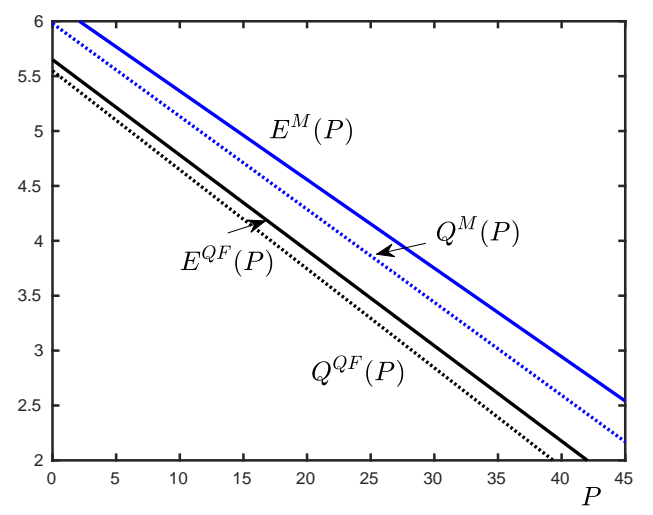

Figure 6b: Emission and standard

Figure 6: Myopic vs. fully dynamic scenarios 